\title{
Epithelial to Mesenchymal Transition of Lung Cancer Cells
}

\author{
Masahiro Seike, Hideaki Mizutani, Junko Sudoh and Akihiko Gemma \\ Division of Pulmonary Medicine, Infection Diseases and Oncology, Graduate School of Medicine, Nippon Medical School
}
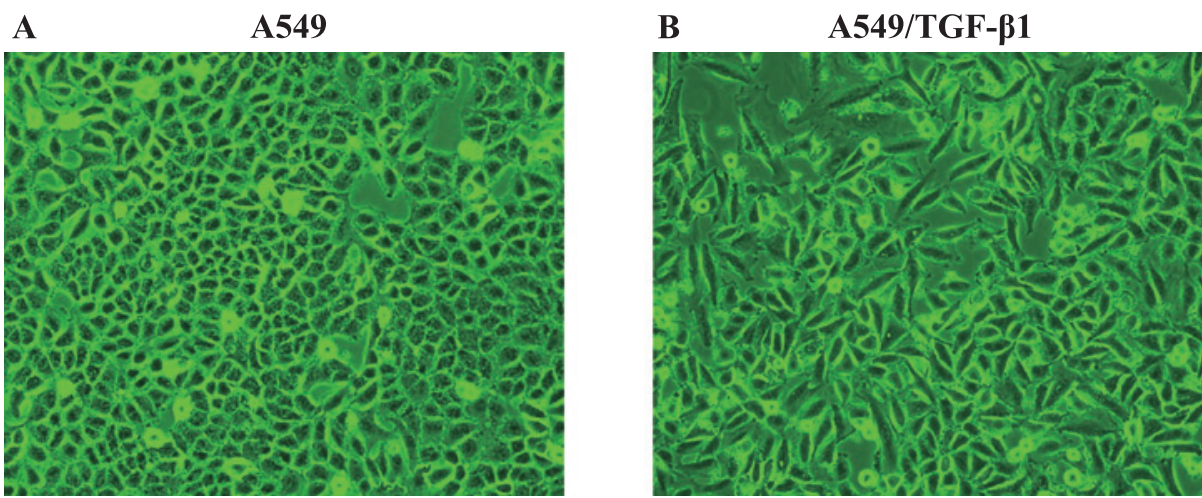

C

A549 A549/TGF- $\beta 1$

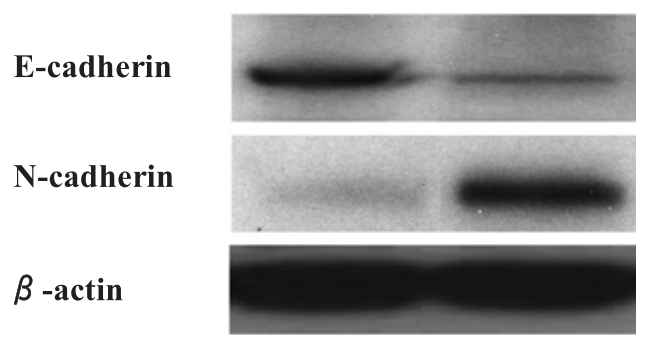

Fig. 1

\begin{abstract}
Epithelial to mesenchymal transition (EMT) is a process by which cells undergo a morphological switch from the epithelial polarized phenotype to the mesenchymal fibroblastoid phenotype and which can be elicited by transforming growth factor- $\beta 1$ (TGF- $\beta 1$ ). EMT has been recognized to play pivotal roles in several diverse processes during embryonic development, chronic inflammation and fibrosis, and the progression of solid tumors, including lung cancer. EMT is a crucial event for lung cancer cells to acquire invasive and metastatic phenotypes. These findings suggest that EMT is a potential target for the chemoprevention and treatment of lung cancer.
\end{abstract}

Fig. 1 TGF- $\beta 1$-stimulated EMT of A549 lung cancer cells A549 cells were treated with $5 \mu \mathrm{g} / \mathrm{mL}$ of TGF- $\beta 1$ for 48 hours and were then designated as A549/ TGF- $\beta 1$ cells. The cells were observed under a light microscope. (A) A549 cells exhibit a classic epithelial morphology. (B) In contrast, A549/TGF- $\beta 1$ cells appeared less uniformly epithelial. (C) Protein expression of EMT markers of A549/TGF- $\beta 1$ cells. Lysates from the A549 and A549/TGF$\beta 1$ cells were subjected to Western blotting. Consistent with EMT, A549/TGF- $\beta 1$ cells lost Ecadherin expression and gained N-cadherin expression.

Correspondence to Masahiro Seike, Department of Pulmonary Medicine/Infection and Oncology, Nippon Medical School, 1-1-5 Sendagi, Bunkyo-ku, Tokyo 113-8603, Japan

E-mail: mseike@nms.ac.jp Journal Website (http://www.nms.ac.jp/jnms/) 